\title{
Nuclear Reactor
}

National Cancer Institute

\section{Source}

National Cancer Institute. Nuclear Reactor. NCI Thesaurus. Code C158342.

A device that enables a controlled, self-sustaining nuclear reaction and release of energy. 